Pacific

Journal of

Mathematics

ON THE ESTIMATE OF THE FIRST EIGENVALUE OF A SUBLAPLACIAN ON A PSEUDOHERMITIAN 3-MANIFOLD

Shu-Cheng Chang And Hung-Lin ChiU 


\title{
ON THE ESTIMATE OF THE FIRST EIGENVALUE OF A SUBLAPLACIAN ON A PSEUDOHERMITIAN 3-MANIFOLD
}

\author{
Shu-Cheng Chang And Hung-Lin Chiu
}

\begin{abstract}
In this paper, we study a lower bound estimate of the first positive eigenvalue of the sublaplacian on a three-dimensional pseudohermitian manifold. S.-Y. $\mathrm{Li}$ and H.-S. Luk derived the lower bound estimate under certain conditions for curvature tensors bounded below by a positive constant. By using the Li-Yau gradient estimate, we are able to get an effective lower bound estimate under a general curvature condition. The key is the discovery of a new CR version of the Bochner formula which involves the CR Paneitz operator.
\end{abstract}

\section{Introduction}

Let $M$ be a closed 3-manifold with an oriented contact structure $\xi$. There always exists a global contact form $\theta$ obtained by patching together local ones with a partition of unity. The characteristic vector field of $\theta$ is the unique vector field $T$ such that $\theta(T)=1$ and $\mathscr{L}_{T} \theta=0$ or $d \theta(T, \cdot)=0$. A CR structure compatible with $\xi$ is a smooth endomorphism $J: \xi \rightarrow \xi$ such that $J^{2}=-$ Id. A pseudohermitian structure compatible with $\xi$ is a CR-structure $J$ compatible with $\xi$ together with a global contact form $\theta$. The CR structure $J$ can extend to $\mathbf{C} \otimes \xi$ and decomposes $\mathbf{C} \otimes \xi$ into the direct sum of $T_{1,0}$ and $T_{0,1}$, which are eigenspaces of $J$ with respect to $i$ and $-i$, respectively.

Let $\left\{T, Z_{1}, Z_{\overline{1}}\right\}$ be a frame of $T M \otimes \mathbf{C}$, where $T$ is the characteristic vector field, $Z_{1}$ is any local frame of $T_{1,0}$, and $Z_{\overline{1}}=\overline{Z_{1}} \in T_{0,1}$. Then $\left\{\theta, \theta^{1}, \theta^{\overline{1}}\right\}$, the coframe dual to $\left\{T, Z_{1}, Z_{\overline{1}}\right\}$, satisfies

$$
d \theta=i h_{1 \overline{1}} \theta^{1} \wedge \theta^{\overline{1}},
$$

for some positive function $h_{1 \overline{1}}$. Actually we can always choose $Z_{1}$ such that $h_{1 \overline{1}}=$ 1 ; hence, throughout this paper, we assume $h_{1 \overline{1}}=1$.

MSC2000: primary 32V05, 32V20; secondary 53C56.

Keywords: eigenvalue, gradient estimate, pseudohermitian manifold, Tanaka-Webster curvature, pseudohermitian torsion, CR Paneitz operator, sublaplacian, Carnot-Carathéodory distance, diameter.

Research supported in part by the NSC of Taiwan. 
The Levi form $\langle,\rangle_{L_{\theta}}$ is the Hermitian form on $T_{1,0}$ defined by

$$
\langle Z, W\rangle_{L_{\theta}}=-i\langle d \theta, Z \wedge \bar{W}\rangle \text {. }
$$

We can extend $\langle,\rangle_{L_{\theta}}$ to $T_{0,1}$ by defining $\langle\bar{Z}, \bar{W}\rangle_{L_{\theta}}=\overline{\langle Z, W\rangle_{L_{\theta}}}$ for all $Z, W \in T_{1,0}$. The Levi form induces naturally a Hermitian form on the dual bundle $\langle,\rangle_{L_{\theta}^{*}}$ of $T_{1,0}$ and hence on all the induced tensor bundles. Integrating the Hermitian form (when acting on sections) over $M$ with respect to the volume form $d V=\theta \wedge d \theta$, we get an inner product on the space of sections of each tensor bundle. We denote the inner product by $\langle$,$\rangle . For example,$

$$
\langle\varphi, \psi\rangle=\int_{M} \varphi \bar{\psi} d V
$$

for functions $\varphi$ and $\psi$.

The pseudohermitian connection of $(J, \theta)$ is the connection $\nabla$ on $T M \otimes \mathbf{C}$ (and extended to tensors) given in terms of a local frame $Z_{1} \in T_{1,0}$ by

$$
\nabla Z_{1}=\theta_{1}^{1} \otimes Z_{1}, \quad \nabla Z_{\overline{1}}=\theta_{\overline{1}}^{\overline{1}} \otimes Z_{\overline{1}}, \quad \nabla T=0,
$$

where $\theta_{1}{ }^{1}$ is the 1 -form uniquely determined by

$$
\begin{aligned}
d \theta^{1} & =\theta^{1} \wedge \theta_{1}{ }^{1}+\theta \wedge \tau^{1}, \\
\tau^{1} & \equiv 0 \quad \bmod \theta^{\overline{1}}, \\
0 & =\theta_{1}{ }^{1}+\theta_{1}{ }^{\overline{1}},
\end{aligned}
$$

where $\tau^{1}$ is the pseudohermitian torsion. Put $\tau^{1}=A^{1}{ }_{\overline{1}} \theta^{\overline{1}}$. The structure equation for the pseudohermitian connection is

$$
d \theta_{1}{ }^{1}=R \theta^{1} \wedge \theta^{\overline{1}}+2 i \operatorname{Im}\left(A_{1, \overline{1}}^{\overline{1}} \theta^{1} \wedge \theta\right),
$$

where $R$ is the Tanaka-Webster curvature.

We will denote components of covariant derivatives with indices preceded by comma; thus we write $A_{1, \overline{1}}{ }_{1} \theta^{1} \wedge \theta$. The indices $\{0,1, \overline{1}\}$ indicate derivatives with respect to $\left\{T, Z_{1}, Z_{\overline{1}}\right\}$. For derivatives of a scalar function, we will often omit the comma, for instance, $\varphi_{1}=Z_{1} \varphi, \varphi_{1 \overline{1}}=Z_{\overline{1}} Z_{1} \varphi-\theta_{1}^{1}\left(Z_{\overline{1}}\right) Z_{1} \varphi, \varphi_{0}=T \varphi$ for a (smooth) function.

For a real function $\varphi$, the subgradient $\nabla_{b}$ is defined by $\nabla_{b} \varphi \in \xi$, and $\left\langle Z, \nabla_{b} \varphi\right\rangle_{L_{\theta}}=$ $d \varphi(Z)$ for all vector fields $Z$ tangent to contact plane. Locally, $\nabla_{b} \varphi=\varphi_{\overline{1}} Z_{1}+\varphi_{1} Z_{\overline{1}}$. We can use the connection to define the subhessian as the complex linear map

$$
\left(\nabla^{H}\right)^{2} \varphi: T_{1,0} \oplus T_{0,1} \rightarrow T_{1,0} \oplus T_{0,1},
$$

and

$$
\left(\nabla^{H}\right)^{2} \varphi(Z)=\nabla_{Z} \nabla_{b} \varphi .
$$


The sublaplacian $\Delta_{b}$ is defined as -1 times the trace of the subhessian, that is, $\Delta_{b} \varphi=-\operatorname{Tr}\left(\left(\nabla^{H}\right)^{2} \varphi\right)=-\left(\varphi_{1 \overline{1}}+\varphi_{\overline{1} 1}\right)$. For all $Z=x^{1} Z_{1} \in T_{1,0}$, define

$$
\begin{aligned}
& \operatorname{Ric}(Z, Z)=R x^{1} x^{\overline{1}}=R|Z|_{L_{\theta}}^{2}, \\
& \operatorname{Tor}(Z, Z)=2 \Re i A_{\overline{1} \overline{1}} x^{\overline{1}} x^{\overline{1}} .
\end{aligned}
$$

Greenleaf [1985] proved the lower bound $(n / n+1) k_{0}$ of the first positive eigenvalue $\lambda_{1}$ of the sublaplacian for a pseudohermitian manifold $M^{2 n+1}$ with $n \geq 3$ under a condition on the Webster curvature and the torsion. Li and Luk [2004] proved the same result for $n=1$ and $n=2$. However, for $n=1$, they needed a condition depending not only on the Webster curvature and the torsion, but also on a covariant derivative of the torsion.

The same result was proved in [Chiu 2006] under a more geometric condition which involved the positivity of the CR Paneitz operator $P_{0}$ (see Section 2 for a definition) with respect to $(J, \theta)$.

Proposition 1.1 [Chiu 2006]. Let $(M, J, \theta)$ be a closed three-dimensional pseudohermitian manifold with nonnegative Paneitz operator $P_{0}$. Suppose that

$$
\operatorname{Ric}_{m}(Z, Z)-\operatorname{Tor}_{m}(Z, Z) \geq k_{0}\langle Z, Z\rangle_{L_{\theta}},
$$

for all $m \in M, Z \in T_{1,0}$, and for some positive constant $k_{0}$. Let $\lambda_{1}$ be the first positive eigenvalue of $\Delta_{b}$. Then

$$
\lambda_{1} \geq \frac{k_{0}}{2}>0
$$

Let $\left(S^{3}, J, \theta\right)$ be a 3 -sphere with the induced CR structure from $\mathbf{C}^{2}$ and the standard contact form $\theta$. One can show that [Chang et al. 2005; Chiu 2006]

$$
\lambda_{1}=\frac{k_{0}}{2} .
$$

Here $k_{0}$ is the positive, constant Webster curvature of $S^{3}$. Thus we get a sharp estimate of $\lambda_{1}$ on the standard sphere $\left(S^{3}, J, \theta\right)$.

Conjecture 1.2. Let $(M, J, \theta)$ be a closed three-dimensional pseudohermitian manifold. Suppose that

$$
\lambda_{1}=\frac{k_{0}}{2} .
$$

We conjecture that $(M, J, \theta)$ is the standard CR 3-sphere due to the theorems of Lichnérowicz [1958] and Obata [1962] in the Riemannian case. In fact, here we have (see the proof of Theorem 1.5)

(i) $\operatorname{Ric}_{m}(Z, Z)-\operatorname{Tor}_{m}(Z, Z)=k_{0}\langle Z, Z\rangle_{L_{\theta}}$,

(ii) $\operatorname{ker}\left(\Delta_{b}-\lambda_{1} I\right) \subset \operatorname{ker} P_{0}$, 
(iii) $\varphi_{11}=0$ for $\varphi \in \operatorname{ker}\left(\Delta_{b}-\lambda_{1} I\right)$.

In this paper, we will try to place a good lower bound on the first positive eigenvalue when the curvature satisfies

$$
\operatorname{Ric}_{m}(Z, Z)-\operatorname{Tor}_{m}(Z, Z) \geq-k_{0}\langle Z, Z\rangle_{L_{\theta}}
$$

for some nonnegative constant $k_{0}$.

Definition 1.3. On a closed pseudohermitian 3-manifold $(M, J, \theta)$, we call the Paneitz operator $P_{0}$ with respect to $(J, \theta)$ essentially positive if there exists a constant $\Lambda>0$ such that

$$
\int_{M} P \varphi \cdot \varphi d \mu \geq \Lambda \int_{M} \varphi^{2} d \mu
$$

for all real $C^{\infty}$ smooth functions $\varphi \in\left(\operatorname{ker} P_{0}\right)^{\perp}$ (that is, those perpendicular to the kernel of $P_{0}$ in the $L^{2}$ norm with respect to the volume form $d \mu=\theta \wedge d \theta$ ).

Remark 1.4. The essential positivity of $P_{0}$ is a CR invariant in the sense that it is independent of the choice of the contact form $\theta$. Actually, if $\widetilde{\theta}=e^{2 \lambda} \theta$ is another contact form, then we have $d \widetilde{V}=\widetilde{\theta} \wedge d \widetilde{\theta}=e^{4 \lambda} \theta \wedge d \theta$ and the transformation law $\widetilde{P}_{0}=e^{-4 \lambda} P_{0}$ of the CR Paneitz operator [Hirachi 1993]. Therefore, we have $\int \widetilde{P}_{0} \varphi \cdot \phi d \widetilde{V}=\int P_{0} \varphi \cdot \phi d V$.

Firstly, by using the same method as in [Chiu 2006], we are able to prove:

Theorem 1.5. Let $(M, J, \theta)$ be a closed three-dimensional pseudohermitian manifold with essentially positive Paneitz operator $P_{0}$. Suppose:

(i) For some nonnegative constant $k_{0}$,

$$
\operatorname{Ric}_{m}(Z, Z)-\operatorname{Tor}_{m}(Z, Z) \geq-k_{0}\langle Z, Z\rangle_{L_{\theta}} .
$$

(ii) $\operatorname{ker}\left(\Delta_{b}-\lambda_{1} I\right) \cap\left(\operatorname{ker} P_{0}\right)^{\perp} \neq \varnothing$.

Then

$$
\lambda_{1} \geq \frac{-k_{0}+\sqrt{k_{0}^{2}+6 \Lambda}}{4}>0 .
$$

However if the torsion is zero, then the corresponding Paneitz operator is essentially positive [Chang et al. 2005]. Therefore,

Corollary 1.6. Let $(M, J, \theta)$ be a closed three-dimensional pseudohermitian manifold. Suppose:

(i) For some nonnegative constant $k_{0}$,

$$
R \geq-k_{0} \quad \text { and } \quad A_{11}=0 .
$$

(ii) $\operatorname{ker}\left(\Delta_{b}-\lambda_{1} I\right) \cap\left(\operatorname{ker} P_{0}\right)^{\perp} \neq \phi$. 
Then

$$
\lambda_{1} \geq \frac{-k_{0}+\sqrt{k_{0}^{2}+6 \Lambda}}{4}>0 .
$$

Definition 1.7. We say that $(M, J)$ has a transversal symmetry if $M$ admits a one-parameter group of $\mathrm{CR}$ automorphisms transverse to the holomorphic tangent bundle.

For example, $(M, J, \theta)$ has a transversal symmetry if $A_{11}=0$. For details, we refer to [Graham and Lee 1988] and [Hirachi 1993].

Definition 1.8. A piecewise smooth curve $\gamma:[0,1] \rightarrow M$ is said to be horizontal if $\gamma^{\prime}(t) \in \xi$ whenever $\gamma^{\prime}(t)$ exists. The length of $\gamma$ is then defined by

$$
l(\gamma)=\int_{0}^{1} d t \sqrt{\left\langle\gamma^{\prime}(t), \gamma^{\prime}(t)\right\rangle_{L_{\theta}}}
$$

The Carnot-Carathéodory distance between two points $p, q \in M$ is

$$
d(p, q)=\inf \left\{l(\gamma) \mid \gamma \in C_{p, q}\right\},
$$

where $C_{p, q}$ is the set of all horizontal curves joining $p$ and $q$. By the Chow connectivity theorem [1939], there always exists a horizontal curve joining $p$ and $q$, so the distance is finite. The diameter $d$ is defined by

$$
d=\sup \{d(p, q) \mid p, q \in M\} .
$$

Note that there is a minimizing geodesic joining $p$ and $q$ so that its length is equal to the distance $d(p, q)$.

Next, by using the Li-Yau gradient estimates [Yau 1975; Li and Yau 1980], we have:

Theorem 1.9. Let $(M, J, \theta)$ be a closed three-dimensional pseudohermitian manifold that has a transversal symmetry. Suppose:

(i) For some nonnegative constant $k_{0}$,

$$
\operatorname{Ric}_{m}(Z, Z)-\operatorname{Tor}_{m}(Z, Z) \geq-k_{0}\langle Z, Z\rangle_{L_{\theta}} .
$$

(ii) $\operatorname{ker}\left(\Delta_{b}-\lambda_{1} I\right) \cap \operatorname{ker} P_{0} \neq \phi$.

Then

$$
\lambda_{1} \geq \frac{\left(1+\sqrt{1+2\left(k_{0}+\tau_{0}\right) d^{2}}\right)}{6 d^{2}} e^{-\left(1+\sqrt{1+2\left(k_{0}+\tau_{0}\right) d^{2}}\right)} .
$$

Here $\tau_{0}=\max \left|A_{11}\right|$ and $d$ is the diameter of $M$.

Corollary 1.10. Let $(M, J, \theta)$ be a closed three-dimensional pseudohermitian manifold. Suppose: 
(i) For some nonnegative constant $k_{0}$,

$$
R \geq-k_{0} \quad \text { and } \quad A_{11}=0 .
$$

(ii) $\operatorname{ker}\left(\Delta_{b}-\lambda_{1} I\right) \cap \operatorname{ker} P_{0} \neq \phi$.

Then

$$
\lambda_{1} \geq \frac{\left(1+\sqrt{1+2 k_{0} d^{2}}\right)}{6 d^{2}} e^{-\left(1+\sqrt{1+2 k_{0} d^{2}}\right)} .
$$

Combining Theorem 1.5 and Theorem 1.9, we can prove:

Theorem 1.11. Let $(M, J, \theta)$ be a closed three-dimensional pseudohermitian manifold which has a transversal symmetry. Suppose:

(i) For some nonnegative constant $k_{0}$,

$$
\operatorname{Ric}_{m}(Z, Z)-\operatorname{Tor}_{m}(Z, Z) \geq-k_{0}\langle Z, Z\rangle_{L_{\theta}} .
$$

(1) $\Delta_{b} \operatorname{ker} P_{0} \subset \operatorname{ker} P_{0}$.

Then

$\lambda_{1} \geq \max \left\{\frac{\left(1+\sqrt{1+2\left(k_{0}+\tau_{0}\right) d^{2}}\right)}{6 d^{2}} e^{-\left(1+\sqrt{1+2\left(k_{0}+\tau_{0}\right) d^{2}}\right)} ; \frac{-k_{0}+\sqrt{k_{0}^{2}+6 \Lambda}}{4}\right\}$.

Here $\tau_{0}=\max \left|A_{11}\right|$ and $d$ is the diameter of $M$.

In particular, if $A_{11}=0$, then $(M, J, \theta)$ has a transversal symmetry and we also have $\Delta_{b} \operatorname{ker} P_{0} \subset \operatorname{ker} P_{0}$. Therefore, as a consequence of Theorem 1.5 and Theorem 1.11,

Corollary 1.12. Let $(M, J, \theta)$ be a closed three-dimensional pseudohermitian manifold with $A_{11}=0$. Suppose

$$
R \geq-k_{0},
$$

for some nonnegative constant $k_{0}$. Then

$$
\lambda_{1} \geq \max \left\{\frac{\left(1+\sqrt{1+2 k_{0} d^{2}}\right)}{6 d^{2}} e^{-\left(1+\sqrt{1+2 k_{0} d^{2}}\right)} ; \frac{-k_{0}+\sqrt{k_{0}^{2}+6 \Lambda}}{4}\right\} .
$$

That is, there is a positive constant $C\left(k_{0}, d, \Lambda\right)$ such that

$$
\lambda_{1} \geq C\left(k_{0}, d, \Lambda\right) .
$$


We briefly describe the methods used in our proofs. In Section 2, we first derive the CR version of Bochner formula which involves the CR Paneitz operator. This formula, involving a term that has no analogue in the Riemannian case, is hard to control. A key step is that we relate this extra term to a third-order operator $P$ that characterizes CR-pluriharmonic functions [Lee 1988]. After integrating by parts, we get the CR Paneitz operator.

Section 3 contains the second crucial step. By using the Li-Yau gradient estimate [Yau 1975; Li and Yau 1980], we are able to prove the main Theorem 1.11.

\section{The Bochner formula and CR Paneitz operator}

We define an operator through

$$
P \varphi=\left(\varphi_{\overline{1}}^{\overline{1}}{ }_{1}+i A_{11} \varphi^{1}\right) \theta^{1}=P \varphi=\left(P_{1} \varphi\right) \theta^{1},
$$

which characterizes the CR-pluriharmonic functions. Here $P_{1} \varphi=\varphi_{1}{ }^{1}{ }_{1}+i A_{11} \varphi^{1}$, and $\bar{P} \varphi=\left(\bar{P}_{1}\right) \theta^{\overline{1}}$ is the conjugate of $P$. Now define $\delta_{b}$ as the divergence operator that takes $(1,0)$-forms to functions by $\delta_{b}\left(\sigma_{1} \theta^{1}\right)=\sigma_{1}{ }^{1}$, and similarly define $\bar{\delta}_{b}$ through $\bar{\delta}_{b}\left(\sigma_{\overline{1}} \theta^{\overline{1}}\right)=\sigma_{\overline{1}}{ }^{\overline{1}}$. The CR Paneitz operator $P_{0}$ is then defined through

$$
P_{0} \varphi=4\left(\delta_{b}(P \varphi)+\bar{\delta}_{b}(\bar{P} \varphi)\right) .
$$

We observe that

$$
\int\left\langle P \varphi+\bar{P} \varphi, d_{b} \varphi\right\rangle_{L_{\theta}^{*}} d V=-\frac{1}{4} \int P_{0} \varphi \cdot \varphi d V
$$

One can check that $P_{0}$ is self-adjoint, that is, $\left\langle P_{0} \varphi, \psi\right\rangle=\left\langle\varphi, P_{0} \psi\right\rangle$ for all smooth functions $\varphi$ and $\psi$. For more details about these operators, read [Lee 1988; Graham and Lee 1988; Hirachi 1993; Gover and Graham 2003; Fefferman and Hirachi 2003].

We first derive the following new CR version of the Bochner formula:

Lemma 2.1. For a real function $\varphi$,

$$
\begin{aligned}
\frac{1}{2} \Delta_{b}\left|\nabla_{b} \varphi\right|^{2}= & -\left|\left(\nabla^{H}\right)^{2} \varphi\right|^{2}+3\left\langle\nabla_{b} \varphi, \nabla_{b} \Delta_{b} \varphi\right\rangle_{L_{\theta}} \\
& -(2 \text { Ric }-3 \text { Tor })\left(\left(\nabla_{b} \varphi\right)_{\mathbf{C}},\left(\nabla_{b} \varphi\right)_{\mathbf{C}}\right) \\
& +4\left\langle P \varphi+\bar{P} \varphi, d_{b} \varphi\right\rangle_{L_{\theta}^{*} .}
\end{aligned}
$$

Here $\left(\nabla_{b} \varphi\right)_{\mathbf{C}}=\varphi_{1} Z_{1}$ is the corresponding complex (1,0)-vector field of $\nabla_{b} \varphi$ and $d_{b} \varphi=\varphi_{1} \theta^{1}+\varphi_{\overline{1}} \theta^{\overline{1}}$. 
Proof. From [Greenleaf 1985], we have for a real function $\varphi$

$$
\begin{aligned}
\Delta_{b}\left|\nabla_{b} \varphi\right|^{2}=-2\left|\left(\nabla^{H}\right)^{2} \varphi\right|^{2}+2\left\langle\nabla_{b} \varphi, \nabla_{b} \Delta_{b} \varphi\right\rangle_{L_{\theta}} \\
-(4 \operatorname{Ric}+2 \operatorname{Tor})\left(\left(\nabla_{b} \varphi\right)_{\mathbf{C}},\left(\nabla_{b} \varphi\right)_{\mathbf{C}}\right)-4\left\langle J \nabla_{b} \varphi, \nabla_{b} \varphi_{0}\right\rangle_{L_{\theta}} .
\end{aligned}
$$

Lemma 2.1 follows from this and

$$
\begin{aligned}
\left\langle J \nabla_{b} \varphi, \nabla_{b} \varphi_{0}\right\rangle_{L_{\theta}}=-\left\langle\nabla_{b} \varphi\right. & \left., \nabla_{b} \Delta_{b} \varphi\right\rangle_{L_{\theta}} \\
& -2 \operatorname{Tor}\left(\left(\nabla_{b} \varphi\right)_{\mathbf{C}},\left(\nabla_{b} \varphi\right)_{\mathbf{C}}\right)-2\left\langle P \varphi+\bar{P} \varphi, d_{b} \varphi\right\rangle_{L_{\theta}^{*}},
\end{aligned}
$$

which we next prove. The commutation relation $i \varphi_{0}=\varphi_{1 \overline{1}}-\varphi_{\overline{1} 1}$ [Lee 1988] gives $\varphi_{1 \overline{1} 1}-\varphi_{\overline{1} 11}=i \varphi_{01}$. Thus

$$
\begin{aligned}
\left\langle J \nabla_{b} \varphi, \nabla_{b} \varphi_{0}\right\rangle_{L_{\theta}} & =i\left(\varphi_{\overline{1}} \varphi_{01}-\varphi_{1} \varphi_{0 \overline{1}}\right) \\
& =\varphi_{\overline{1}}\left(\varphi_{1 \overline{1} 1}-\varphi_{\overline{1} 11}\right)+\varphi_{1}\left(\varphi_{\overline{1} 1 \overline{1}}-\varphi_{1 \overline{1} \overline{1}}\right) .
\end{aligned}
$$

On the other hand,

$$
\begin{aligned}
\left\langle\nabla_{b} \varphi, \nabla_{b} \Delta_{b} \varphi\right\rangle_{L_{\theta}} & =\varphi_{\overline{1}}\left(\Delta_{b} \varphi\right)_{1}+\varphi_{1}\left(\Delta_{b} \varphi\right)_{\overline{1}} \\
& =-\varphi_{\overline{1}}\left(\varphi_{1 \overline{1} 1}+\varphi_{\overline{1} 11}\right)-\varphi_{1}\left(\varphi_{\overline{1} 1 \overline{1}}+\varphi_{1 \overline{1} \overline{1}}\right) .
\end{aligned}
$$

It follows from (4) and (5) that

$$
\begin{aligned}
\left\langle J \nabla_{b} \varphi, \nabla_{b} \varphi_{0}\right\rangle_{L_{\theta}}+\left\langle\nabla_{b} \varphi\right. & \left., \nabla_{b} \Delta_{b} \varphi\right\rangle_{L_{\theta}}=-2 \varphi_{\overline{1}}^{-} \varphi_{\overline{1} 11}-2 \varphi_{1} \varphi_{1 \overline{1} \overline{1}} \\
& =-2 \varphi_{\overline{1}}\left(P_{1} \varphi-i A_{11} \varphi_{\overline{1}}\right)-2 \varphi_{1}\left(\bar{P}_{1} \varphi+i A_{\overline{1} \overline{1}} \varphi_{1}\right) \\
& =-2 \operatorname{Tor}\left(\left(\nabla_{b} \varphi\right)_{\mathbb{C}},\left(\nabla_{b} \varphi\right)_{\mathbb{C}}\right)-2\left\langle P \varphi+\bar{P} \varphi, d_{b} \varphi\right\rangle_{L_{\theta}^{*}} .
\end{aligned}
$$

Proof of Theorem 1.5. Let $\varphi \in\left(\operatorname{ker} P_{0}\right)^{\perp}$ be an eigenfunction of $\Delta_{b}$ having the first positive eigenvalue $\lambda_{1}$. By definition,

$$
\int_{M} \varphi P_{0} \varphi d V \geq \Lambda \int_{M} \varphi^{2} d V
$$

By integrating (2), (3) and using (1), we have

$$
\begin{aligned}
\int\left|\left(\nabla^{H}\right)^{2} \varphi\right|_{L_{\theta}}^{2} d V=\int\left(\Delta_{b} \varphi\right)^{2} d V & +2 \int \varphi_{0}^{2} d V \\
& -\int(2 \operatorname{Ric}+\operatorname{Tor})\left(\left(\nabla_{b} \varphi\right)_{\mathbb{C}},\left(\nabla_{b} \varphi\right)_{\mathbb{C}}\right) d V
\end{aligned}
$$

and

(8) $\int \varphi_{0}^{2} d V=\int\left(\Delta_{b} \varphi\right)^{2} d V+2 \int \operatorname{Tor}\left(\left(\nabla_{b} \varphi\right)_{\mathbb{C}},\left(\nabla_{b} \varphi\right)_{\mathbb{C}}\right) d V-\frac{1}{2} \int P_{0} \varphi \cdot \varphi d V$.

On the other hand, it is easy to verify that

$$
\left|\left(\nabla^{H}\right)^{2} \varphi\right|_{L_{\theta}}^{2}=2\left|\varphi_{11}\right|^{2}+\frac{1}{2}\left(\Delta_{b} \varphi\right)^{2}+\frac{1}{2} \varphi_{0}^{2} .
$$


Substituting this into the left hand of (7) and combining with (8), we get

$$
\begin{aligned}
2 \int\left|\varphi_{11}\right|^{2} d V=2 \int\left(\Delta_{b} \varphi\right)^{2} d V-2 \int(\operatorname{Ric}-\operatorname{Tor})\left(\left(\nabla_{b} \varphi\right)_{\mathbb{C}},\right. & \left.\left(\nabla_{b} \varphi\right)_{\mathbb{C}}\right) d V \\
& -\frac{3}{4} \int P_{0} \varphi \cdot \varphi d V
\end{aligned}
$$

By combining with (6), we get

$$
\begin{aligned}
0 & \geq-2 \int\left(\Delta_{b} \varphi\right)^{2} d V+2 \int(\text { Ric }- \text { Tor })\left(\left(\nabla_{b} \varphi\right)_{\mathbb{C}},\left(\nabla_{b} \varphi\right)_{\mathbb{C}}\right) d V+\frac{3}{4} \int P_{0} \varphi \cdot \varphi d V \\
& \geq \int-2 \lambda_{1}\left|\nabla_{b} \varphi\right|_{L_{\theta}}^{2} d V-\int k_{0}\left|\nabla_{b} \varphi\right|_{L_{\theta}}^{2} d V+\int \frac{3 \Lambda}{4} \varphi^{2} d V \\
& =\int\left(-2 \lambda_{1}-k_{0}+\frac{3 \Lambda}{4 \lambda_{1}}\right)\left|\nabla_{b} \varphi\right|_{L_{\theta}}^{2} d V .
\end{aligned}
$$

This holds if and only if

$$
-2 \lambda_{1}-k_{0}+\frac{3 \Lambda}{4 \lambda_{1}} \leq 0
$$

and Theorem 1.5 follows immediately.

\section{The Li-Yau gradient estimate}

Let $(M, J, \theta)$ be a closed three-dimensional pseudohermitian manifold. In the case that $\operatorname{ker}\left(\Delta_{b}-\lambda_{1} I\right) \cap\left(\operatorname{ker} P_{0}\right) \neq \phi$, then, by using the so-called Li-Yau gradient estimate [Yau 1975; Li and Yau 1980], one can place a lower bound on the positive first eigenvalue of a sublaplacian $\Delta_{b}$.

Lemma 3.1. Let $\varphi=\ln f$ for $f>0$. Then

$$
\begin{aligned}
4\left\langle P \varphi+\bar{P} \varphi, d_{b} \varphi\right\rangle_{L_{\theta}^{*}}= & 4 \frac{\left\langle P f+\bar{P} f, d_{b} f\right\rangle_{L_{\theta}^{*}}}{f^{2}}-4\left\langle\nabla_{b} \varphi, \nabla_{b}\left|\nabla_{b} \varphi\right|^{2}\right\rangle_{L_{\theta}} \\
& +2 \frac{\Delta_{b} f}{f}\left|\nabla_{b} \varphi\right|^{2} .
\end{aligned}
$$

Proof. Let $Q(x)=\left|\nabla_{b} \varphi\right|^{2}(x)$. We compute

$$
\begin{aligned}
\nabla_{b} Q & =Q_{\overline{1}} Z_{1}+Q_{1} Z_{\overline{1}}=2 \nabla_{b}\left(\varphi_{1} \varphi_{\overline{1}}\right) \\
& =\frac{f^{2} f_{1} f_{\overline{11}}+f^{2} f_{\overline{1}} f_{1 \overline{1}}-2 f f_{\overline{1}}^{2} f_{1}}{f^{4}} Z_{1}+\text { complex conjugate. }
\end{aligned}
$$


It follows that

$$
\begin{aligned}
P_{1} \varphi=\varphi_{\overline{1} 11}+i A_{11} \varphi_{\overline{1}} & =\frac{f^{3} f_{\overline{1} 11}-f^{2} f_{\overline{1}} f_{11}-2 f^{2} f_{1} f_{\overline{1} 1}+2 f f_{1}^{2} f_{\overline{1}}}{f^{4}}+i A_{11} \frac{f_{\overline{1}}}{f} \\
& =\frac{P_{1} f}{f}-Q_{1}-\frac{f_{1} f_{\overline{1} 1}}{f^{2}}=\frac{P_{1} f}{f}-Q_{1}-\varphi_{1} \frac{f_{\overline{1} 1}}{f} .
\end{aligned}
$$

Thus

$$
\begin{aligned}
& 4\left\langle P \varphi+\bar{P} \varphi, d_{b} \varphi\right\rangle_{L_{\theta}^{*}}=4\left\langle\left(P_{1} \varphi\right) \theta^{1}+\left(\bar{P}_{1} \varphi\right) \theta^{\overline{1}}, \varphi_{1} \theta^{1}+\varphi_{\overline{1}} \theta^{\overline{1}}\right\rangle_{L_{\theta}^{*}} \\
& =4\left[\left(P_{1} \varphi\right) \varphi_{\overline{1}}+\left(\bar{P}_{1} \varphi\right) \varphi_{1}\right]=4\left(\frac{P_{1} f}{f}-Q_{1}-\varphi_{1} \frac{f_{\overline{1} 1}}{f}\right) \varphi_{\overline{1}}+\text { complex conjugate } \\
& =4 \frac{\left\langle P f+\bar{P} f, d_{b} f\right\rangle_{L_{\theta}^{*}}}{f^{2}}-4\left\langle\nabla_{b} \varphi, \nabla_{b}\left|\nabla_{b} \varphi\right|^{2}\right\rangle+2\left(\frac{\Delta_{b} f}{f}\left|\nabla_{b} \varphi\right|^{2}\right) .
\end{aligned}
$$

This implies the lemma.

The next lemma will ready us to show Theorem 1.9.

Lemma 3.2 [Graham and Lee 1988; Hirachi 1993]. Let $(M, J, \theta)$ be a closed three-dimensional pseudohermitian manifold with a transversal symmetry, and let $\theta$ be any pseudohermitian structure on $M$. Then a smooth real-valued function $f$ satisfies $P_{0} f=0$ on $M$ if and only if $P_{1} f=0$ on $M$, that is, $f$ is CR-pluriharmonic.

Proof of Theorem 1.9. Let $f$ be an eigenfunction of $\Delta_{b}$ with eigenvalue $\lambda_{1}$, that is, $\Delta_{b} f=\lambda_{1} f$. Also suppose $P_{0} f=0$. Since

$$
\lambda_{1} \int_{M} f=\int_{M} \Delta_{b} f=0
$$

$f$ must change sign. We may normalize $f$ to satisfy $\min f=-1$ and $\max f \leq 1$. Let us consider the function $\varphi=\ln (a+f)$, for some constant $a>1$. Then the function $\varphi$ satisfies

$$
\begin{aligned}
\Delta_{b} \varphi & =\frac{\Delta_{b} f}{a+f}-\left\langle\nabla_{b}\left(\frac{1}{a+f}\right), \nabla_{b}(a+f)\right\rangle_{L_{\theta}} \\
& =\frac{\Delta_{b} f}{a+f}+\frac{\left|\nabla_{b} f\right|^{2}}{(a+f)^{2}}=\frac{\lambda_{1} f}{a+f}+\left|\nabla_{b} \varphi\right|^{2} .
\end{aligned}
$$

Since $\left|\left(\nabla^{\xi}\right)^{2} \varphi\right|_{L_{\theta}}^{2}=2\left|\varphi_{11}\right|^{2}+\frac{1}{2}\left(\Delta_{b} \varphi\right)^{2}+\frac{1}{2} \varphi_{0}^{2}$, we have

$$
-\left|\left(\nabla^{\xi}\right)^{2} \varphi\right|_{L_{\theta}}^{2} \leq-\frac{1}{2}\left(\Delta_{b} \varphi\right)^{2} \leq-\frac{1}{2}\left|\nabla_{b} \varphi\right|^{4}-\frac{\lambda_{1} f}{a+f}\left|\nabla_{b} \varphi\right|^{2}
$$


On the other hand, we have

$$
\begin{aligned}
\left\langle\nabla_{b} \varphi, \nabla_{b} \Delta_{b} \varphi\right\rangle_{L_{\theta}} & =\left\langle\nabla_{b} \varphi, \nabla_{b}\left(\frac{\lambda_{1} f}{a+f}+\left|\nabla_{b} \varphi\right|^{2}\right)\right\rangle_{L_{\theta}} \\
& =\left\langle\nabla_{b} \varphi, \nabla_{b}\left|\nabla_{b} \varphi\right|^{2}\right\rangle_{L_{\theta}}+\frac{\lambda_{1} a}{a+f}\left|\nabla_{b} \varphi\right|^{2} .
\end{aligned}
$$

Because $\operatorname{Ric}_{m}(Z, Z)-\operatorname{Tor}_{m}(Z, Z) \geq-k_{0}\langle Z, Z\rangle_{L_{\theta}}$, we have

$$
2 \operatorname{Ric}_{m}(Z, Z)-2 \operatorname{Tor}_{m}(Z, Z) \geq-2 k_{0}\langle Z, Z\rangle_{L_{\theta}} \text {. }
$$

On the other hand, put $\tau_{0}=\max \left|A_{11}\right|$. Then from

$$
-2\left|A_{11}\right|\langle Z, Z\rangle_{L_{\theta}} \leq-\operatorname{Tor}(Z, Z) \leq 2\left|A_{11}\right|\langle Z, Z\rangle_{L_{\theta}},
$$

we have

$$
-2 \tau_{0}\langle Z, Z\rangle_{L_{\theta}} \leq-\operatorname{Tor}(Z, Z) \leq 2 \tau_{0}\langle Z, Z\rangle_{L_{\theta}} .
$$

Combining (9) and (10), one has

$$
2 \operatorname{Ric}_{m}(Z, Z)-3 \operatorname{Tor}_{m}(Z, Z) \geq-2\left(k_{0}+\tau_{0}\right)\langle Z, Z\rangle_{L_{\theta}} .
$$

Now we define $Q(x)=\left|\nabla_{b} \varphi\right|^{2}$. Then, by Lemma 2.1 and Lemma 3.1, we see that the sublaplacian satisfies

$$
\begin{aligned}
\frac{1}{2} \Delta_{b} Q+\left\langle\nabla_{b} \varphi, \nabla_{b} Q\right\rangle & \leq-\frac{1}{2} Q^{2}-\left(\lambda_{1}-\frac{2\left(k_{0}+\tau_{0}\right)}{2}-\frac{4 \lambda_{1} a}{a+f}-\frac{2 \lambda_{1} f}{a+f}\right) Q \\
& \leq-\frac{1}{2} Q^{2}-\left(\lambda_{1}-\frac{2\left(k_{0}+\tau_{0}\right)}{2}-\frac{6 \lambda_{1} a}{a-1}\right) Q .
\end{aligned}
$$

If $x_{0} \in M$ is a point where $Q$ achieves its maximum, we have

$$
0 \leq \frac{1}{2} \Delta_{b} Q\left(x_{0}\right)+\left\langle\nabla_{b} \varphi, \nabla_{b} Q\right\rangle\left(x_{0}\right) .
$$

Hence

$$
\frac{1}{2} Q^{2}\left(x_{0}\right)+\left(\lambda_{1}-\frac{2\left(k_{0}+\tau_{0}\right)}{2}-\frac{6 \lambda_{1} a}{a-1}\right) Q\left(x_{0}\right) \leq 0
$$

which implies that

$$
Q(x) \leq Q\left(x_{0}\right) \leq-2\left(\lambda_{1}-\frac{2\left(k_{0}+\tau_{0}\right)}{2}-\frac{6 \lambda_{1} a}{a-1}\right) \leq \frac{12 a}{a-1} \lambda_{1}+2\left(k_{0}+\tau_{0}\right),
$$


for all $x \in M$. Integrating $Q^{\frac{1}{2}}=\left|\nabla_{b} \varphi\right|=\left|\nabla_{b} \ln (a+f)\right|$ along a minimal horizontal geodesic $\gamma$ joining the points at which $f=-1$ and $f=\max f$, it follows that

$$
\begin{aligned}
\ln \frac{a}{a-1} & \leq \ln \left(\frac{a+\max f}{a-1}\right)=\ln (a+\max f)-\ln (a-1) \\
& \leq \int_{\gamma}\left|\nabla_{b} \ln (a+f)\right| \leq d \sqrt{\frac{12 a}{a-1} \lambda_{1}+2\left(k_{0}+\tau_{0}\right)},
\end{aligned}
$$

for all $a>1$. Setting $t=(a-1) / a$, we have

$$
12 \lambda_{1} \geq\left(\frac{1}{d^{2}}\left(\ln \frac{1}{t}\right)^{2}-2\left(k_{0}+\tau_{0}\right)\right) t
$$

for all $0<t<1$. Maximizing the right hand side as a function of $t$ by setting $t=\exp \left(-1-\sqrt{1+2\left(k_{0}+\tau_{0}\right) d^{2}}\right)$, we obtain the estimate

$$
\begin{aligned}
\lambda_{1} & \geq \frac{1}{12}\left(\frac{\left(1+\sqrt{1+2\left(k_{0}+\tau_{0}\right) d^{2}}\right)^{2}}{d^{2}}-2\left(k_{0}+\tau_{0}\right)\right) e^{\left(-1-\sqrt{1+2\left(k_{0}+\tau_{0}\right) d^{2}}\right)} \\
& =\frac{\left(1+\sqrt{1+2\left(k_{0}+\tau_{0}\right) d^{2}}\right)}{6 d^{2}} e^{\left(-1-\sqrt{1+2\left(k_{0}+\tau_{0}\right) d^{2}}\right)} .
\end{aligned}
$$

Proof of Theorem 1.11. If $(M, J, \theta)$ is a closed three-dimensional pseudohermitian manifold that has a transversal symmetry, then there exists a torsion free pseudohermitian contact structure $\widetilde{\theta}=e^{2 f} \theta$ for some real smooth function $f$. Therefore $\widetilde{P}_{0}$, the CR Paneitz operator with respect to $\widetilde{\theta}$, is essentially positive. But $\widetilde{P}_{0}=e^{-4 f}$ $P_{0}$. It follows that $P_{0}$ is essentially positive.

On the other hand, suppose that the CR Paneitz operator $P_{0}$ and the sublaplacian $\Delta_{b}$ satisfy $\Delta_{b}\left(\operatorname{ker} P_{0}\right) \subset \operatorname{ker} P_{0}$. Hence we have the following decomposition (see [Chang et al. 2005, Section 5] for details):

$$
\operatorname{ker}\left(\Delta_{b}-\lambda_{1} I\right)=E_{K} \oplus_{P_{0}} E_{K}^{\perp}
$$

where $E_{K} \subset \operatorname{ker} P_{0}$ and $E_{K}{ }^{\perp} \subset\left(\operatorname{ker} P_{0}\right)^{\perp}$.

Let $f$ be an eigenfunction of $\Delta_{b}$ with respect to the first positive eigenvalue $\lambda_{1}$. $P_{0}$ decomposes $f$ as

$$
f=f^{\perp} \oplus f_{\mathrm{ker}},
$$

whence

$$
\Delta_{b} f^{\perp}=\lambda_{1} f^{\perp} \quad \text { and } \quad \Delta_{b} f_{\mathrm{ker}}=\lambda_{1} f_{\mathrm{ker}} .
$$

Theorem 1.11 then follows directly from Theorem 1.5 and Theorem 1.9. 


\section{Acknowledgments}

The first author would like to express his thanks to Professor S.-T. Yau for constant encouragement and Professor J.-P. Wang for valuable discussions during his visit at NCTS, Hsinchu, Taiwan.

\section{References}

[Chang et al. 2005] S.-C. Chang, J.-H. Cheng, and H.-L. C. Chiu, "The fourth-order Q-curvature flow on a CR 3-manifold", preprint, 2005, Available at http://www.arxiv.org/abs/math/0510494.

[Chiu 2006] H.-L. Chiu, "The sharp lower bound for the first positive eigenvalue of the sublaplacian on a pseudohermitian 3-manifold", Ann. Global Anal. Geom. 30:1 (2006), 81-96. MR 2007j:58034 Zbl 1098.32017

[Chow 1939] W.-L. Chow, "Über Systeme von linearen partiellen Differentialgleichungen erster Ordnung”, Math. Ann. 117 (1939), 98-105. MR 1,313d Zbl 0022.02304

[Fefferman and Hirachi 2003] C. Fefferman and K. Hirachi, "Ambient metric construction of $Q$ curvature in conformal and CR geometries", Math. Res. Lett. 10:5-6 (2003), 819-831. MR 2005d: 53044 Zbl 02064736

[Gover and Graham 2003] A. Gover and C. Graham, " $C R$ Invariant powers of the sub-Laplacian”, (2003).

[Graham and Lee 1988] C. R. Graham and J. M. Lee, "Smooth solutions of degenerate Laplacians on strictly pseudoconvex domains", Duke Math. J. 57:3 (1988), 697-720. MR 90c:32031 Zbl 0699.35112

[Greenleaf 1985] A. Greenleaf, "The first eigenvalue of a sub-Laplacian on a pseudo-Hermitian manifold”, Communications in Partial Differential Equations 10:2 (1985), 191-217. MR 86f:58157 Zbl 0563.58034

[Hirachi 1993] K. Hirachi, "Scalar pseudo-Hermitian invariants and the Szegő kernel on threedimensional CR manifolds", pp. 67-76 in Complex geometry (Osaka, 1990), edited by G. Komatsu and Y. Sakane, Lecture Notes in Pure and Appl. Math. 143, Dekker, New York, 1993. MR 93k:32036 Zbl 0805.32014

[Lee 1988] J. M. Lee, "Pseudo-Einstein structures on CR manifolds", Amer. J. Math. 110:1 (1988), 157-178. MR 89f:32034 Zbl 0683:32019

[Li and Luk 2004] S.-Y. Li and H.-S. Luk, "The sharp lower bound for the first positive eigenvalue of a sub-Laplacian on a pseudo-Hermitian manifold", Proc. Amer. Math. Soc. 132:3 (2004), 789-798. MR 2005c:58056 Zbl 1041:32024

[Li and Yau 1980] P. Li and S. T. Yau, "Estimates of eigenvalues of a compact Riemannian manifold”, pp. 205-239 in Geometry of the Laplace operator (Honolulu, 1979), edited by R. Osserman and A. Weinstein, Proc. Sympos. Pure Math. 36, Amer. Math. Soc., Providence, R.I., 1980. MR 81i:58050 Zbl 0441.58014

[Lichnérowicz 1958] A. Lichnérowicz, Géométrie des groupes de transformations, Travaux et recherches mathématiques 3, Dunod, Paris, 1958. Zbl 0096.16001

[Obata 1962] M. Obata, "Certain conditions for a Riemannian manifold to be isometric with a sphere”, J. Math. Soc. Japan 14 (1962), 333-340. MR 25 \#5479 Zbl 0115:39302

[Yau 1975] S. T. Yau, "Harmonic functions on complete Riemannian manifolds", Comm. Pure Appl. Math. 28 (1975), 201-228. MR 55 \#4042 Zbl 0291:31002 
Received April 25, 2006.

SHU-CHENG CHANG

DEPARTMENT OF MATHEMATICS

NATIONAL TSING HUA UNIVERSITY

HSINCHU 30013

TAIWAN

scchang@math.nthu.edu.tw

HUNG-LIN CHIU

DePARTMENT OF APPLIEd MATHEMATICS

National CEnTRAL University

CHUNG-Li 32054

TAIWAN

hlchiu@math.ncu.edu.tw 